\title{
Discovery of LAMP-2A as potential biomarkers for glioblastoma development by modulating apoptosis through $\mathrm{N}-\mathrm{CoR}$ degradation
}

\author{
Yongjie Wang ${ }^{1}$, Buyi Zhang ${ }^{2}$, Jianli Wang ${ }^{1}$, Haijian Wu ${ }^{1}$, Shenbin $\mathrm{Xu}^{1}$, Jianmin Zhang ${ }^{1 *}$ and Lin Wang ${ }^{1 *}$ (D)
}

\begin{abstract}
Background: Lysosome-associated membrane protein type 2A (LAMP-2A) is the key component of chaperonemediated autophagy (CMA), a cargo-selective lysosomal degradation pathway. Aberrant LAMP-2A expression and CMA activation have been demonstrated in various human malignancies. The study focusing on the intrinsic role of LAMP-2A and CMA in glioblastoma (GBM), and downstream mechanism could provide valuable insight into the pathogenesis and novel therapeutic modality of GBM.
\end{abstract}

Methods: The levels of LAMP-2A, nuclear receptor co-repressor (N-CoR), unfolded protein response (UPR) and apoptosis were examined in clinical samples. LAMP-2A siRNA and shRNA were constructed to manipulate CMA activation. The role of CMA and downstream mechanism through degradation of N-COR and arresting UPR mediated apoptosis were explored in GBM cells and nude mouse xenograft model.

Results: Elevated LAMP-2A and associated decreased N-CoR expression were observed in GBM as compared with peritumoral region and low-grade glioma. Inhibited UPR and apoptosis were observed in GBM with high LAMP-2A expression. In vitro study demonstrated co-localization and interaction between LAMP-2A and N-CoR. LAMP-2A silencing up-regulated N-CoR and aroused UPR pathway, leading to apoptosis, while N-CoR silencing led to an opposite result. In vivo study further confirmed that LAMP-2A inhibition arrested tumor growth by promoting apoptosis.

Conclusions: Our results demonstrated the central role of CMA in mediating N-CoR degradation and protecting GBM cells against UPR and apoptosis, and provided evidence of LAMP-2A as potential biomarker. Further research focusing on CMA with other tumorigenic process is needed and selective modulators of LAMP-2A remain to be investigated to provide a novel therapeutic strategy for GBM.

Keywords: Glioblastoma, LAMP-2A, Chaperone-mediated autophagy, Nuclear receptor co-repressor, Unfolded protein response, Apoptosis

\footnotetext{
*Correspondence: zjm135@zju.edu.cn; dr_wang@zju.edu.cn

1 Department of Neurosurgery, 2Nd Affiliated Hospital, School

of Medicine, Zhejiang University, 88\# Jiefang Road, Hangzhou 310009, Zhejiang, China

Full list of author information is available at the end of the article
}

\section{Background}

Glioblastoma (GBM) is the most prevalent and aggressive primary brain tumor. Even with maximal safe resection, standard radiotherapy and temozolomide chemotherapy, the median survival is only 14.6 months [1]. Although advances in molecular profiling promote the identification of various potential biomarkers and treatment original author(s) and the source, provide a link to the Creative Commons licence, and indicate if changes were made. The images or other third party material in this article are included in the article's Creative Commons licence, unless indicated otherwise in a credit line to the material. If material is not included in the article's Creative Commons licence and your intended use is not permitted by statutory regulation or exceeds the permitted use, you will need to obtain permission directly from the copyright holder. To view a copy of this licence, visit http://creativecommons.org/licenses/by/4.0/. The Creative Commons Public Domain Dedication waiver (http://creativeco mmons.org/publicdomain/zero/1.0/) applies to the data made available in this article, unless otherwise stated in a credit line to the data. 
targets, including the famous monoclonal anti-VEGFA antibody bevacizumab, there has not been significant improvement in survival of GBM $[2,3]$. Therefore insights into the potential biomarkers and molecular mechanism for GBM development and progression still remain important in seeking new effective therapeutic targets.

As an evolutionarily conserved proteolytic pathway, autophagy maintains intracellular stasis through lysosome-mediated degradation [4]. The pathophysiological pathways of commonly known macroautophagy and microautophagy have been well described. Chaperonemediated autophagy (CMA), on the contrary, is a highly selective degradation process targeting KFERQ-likemotif-bearing proteins, during which the substrates are recognized by heat shock cognate $71 \mathrm{kDa}$ protein (Hsc70) and co-chaperones, and delivered to the surface of the lysosome where they bind to lysosomal associated membrane protein 2A (LAMP-2A) and undergo unfolding, and are finally translocated into the lysosomes where they are rapidly degraded [5-7]. The process is crucial for cellular homeostasis and adaptation to various forms of stress [8]. The specific characteristics of CMA lead to the researches that proved the linkage of deregulated CMA to the progression of various diseases [9]. Abnormally up-regulated LAMP-2A, as an indicator of activated CMA, has been demonstrated in a variety of human malignancies [10, 11]. Recent published papers identified the role of LAMP-2A in modulating GBM-pericytes interaction and temozolomide resistance via CMA [1214]. However, the intrinsic function of LAMP-2A and CMA in GBM development and progression has been poorly elucidated.

Nuclear receptor co-repressor (N-CoR) is a vital component of a multi-protein repressor complex that is normally confined to nucleus and essential for the function of tumor suppressor proteins [15]. Misfolded N-CoR is transported out of nucleus and accumulated in the cytoplasm during malignant transformation of normal cells [16]. However, exceedingly intracytoplasmic aggregation of N-CoR activates unfolded protein response (UPR) and fires apoptosis cascade as means of self-destruction to stop canceration, via various stress sensing proteins anchored on endoplasmic reticulum (ER), including pancreatic ER kinase (PERK)-C/EBP homologous protein (CHOP) and inositol requiring enzyme 1 (IRE1)-JUN N-terminal kinase (JNK) pathway [16-18]. How cancer cells survive from stress caused by harmful protein accumulation remains unclear. In acute promyelocytic leukemia and non-small cell lung cancer, CMA is found to mediate misfolded N-CoR clearance to promote cancer cell survival $[19,20]$. In GBM, although correlation between low level of $\mathrm{N}-\mathrm{CoR}$ and tumor development and aggressiveness has been found [21, 22], the upstream regulatory mechanism is unknown.

Here we explored the expression of LAMP-2A in GBM and whether CMA sheltered tumor cells away from the accumulative stress from $\mathrm{N}$-CoR. In this report, we first proved elevated LAMP-2A and decreased N-CoR expression in GBM clinical samples, and demonstrated that LAMP-2A targeted N-CoR for CMA degradation and as a result promoted the proliferation of GBM by inhibiting UPR mediated apoptosis in GBM cell and nude mice xenograft models. The findings provide support for LAMP$2 \mathrm{~A}$ as potential biomarkers for GBM and valuable insight into the pathogenesis and novel therapeutic modality of GBM.

\section{Methods}

\section{Clinical samples}

A total of 24 tissue specimens were obtained from low grade glioma (LGG, WHO II, $\mathrm{n}=8$ ), tumor center $(\mathrm{n}=8)$ and peri-tumor edema zone (PTEZ, $\mathrm{n}=8$ ) of GBM in the Second Affiliated Hospital of Zhejiang University School of Medicine from January 2017 to January 2018. Glioma was diagnosed according to the 2016 WHO Classification of Tumors of the Central Nervous System. Written informed consents were obtained from all the patients receiving surgical interventions. The study was approved by the Ethical Committee of the Second Affiliated Hospital of Zhejiang University School of Medicine. The clinical samples were used for quantitative polymerase chain reaction (qPCR), western blot (WB) and immunohistochemistry (IHC) analyses.

\section{Cell cultures and cell transfection}

The glioma cell line U87-MG was purchased from the Cell Library of the Chinese Academy of Sciences (Shanghai, China). U87-MG was maintained in Dulbecco's modified Eagle's medium (DMEM; Gibco, Carlsbad, CA, USA) with $10 \%$ fetal bovine serum (FBS, Gibco) and 100 $\mathrm{U} / \mathrm{ml}$ penicillin-streptomycin (Gibco). Starvation experiment was performed in DMEM medium without FBS for $24 \mathrm{~h}$ before being harvested for further analysis. For selection of efficient LAMP-2A and N-CoR siRNA, cells were cultured in 6-well plate at density of $5 \times 10^{5} / \mathrm{ml}$ until confluence of $60-70 \%$. The medium was replaced by Opti-MEM (Invitrogen, Grand Island, NY, USA). The following siRNAs were designed and transfected according to the Lipofectamine 2000 protocol (Lipo2000, Invitrogen): Negative control of LAMP-2A, $5^{\prime} \rightarrow 3^{\prime}$-UUCUCC GAACGUGUCACGU; LAMP-2A siRNA1, $5^{\prime} \rightarrow 3^{\prime}$-CUG GGAUGUUCUUGUACAA; LAMP-2A siRNA2, $5^{\prime} \rightarrow 3^{\prime}$-GCUCUACUUAGACUCAAUA; LAMP-2A siRNA 3, 5' $\rightarrow 3^{\prime}$-GCCUUGGGCUUCCUUAUAA; Negative control of $\mathrm{N}$-CoR, $5^{\prime} \rightarrow 3^{\prime}$-UUCUCCGAACGU 
GUCACGU; N-CoR siRNA1, $5^{\prime} \rightarrow 3^{\prime}$-GACGAGUCA AGUUCAUUAA; N-CoR siRNA2, $5^{\prime} \rightarrow 3^{\prime}$-GACCCU AUGAAAGUGUAUA; LAMP-2A siRNA $3,5^{\prime} \rightarrow 3^{\prime}$-CCU CGUCAGAAGGAAUUAU. The cells were harvested after $72 \mathrm{~h}$ post-transfection for mRNA quantification. The siRNA with best inhibitory effect was utilized for in vitro study and constructing LAMP-2A shRNA lentivirus. To study the involvement of apoptosis, U87-MG cells were pretreated with pan-caspase inhibitor ZvaD (MedChemExpress, NJ, USA) at concentration of $40 \mu \mathrm{m}$ for 30 min before being transfected with siRNAs.

\section{Constructing lentivirus-mediated LAMP-2A shRNA}

The pLKO.1-shLAMP-2A and control plasmid were designed and cloned, together with package plasmids including psPAX2 and PMD2G. These lentivirus-based constructs were co-transfected into cultured $293 \mathrm{~T}$ cells. The replication-deficient lentiviral particles were packaged and collected $72 \mathrm{~h}$ later for in vivo study.

\section{Nude mouse xenograft studies}

The study was approved by the Institutional Animal Care and Use Committee of The Second Affiliated Hospital of Zhejiang University, School of Medicine. The male BALB/c nude mice (average weight $20 \mathrm{~g}$; 6-8 weeks old) were purchased from Experimental Animal Laboratories (SLAC, Shanghai, China). The mice were cultivated in a specific pathogen-free room at $20{ }^{\circ} \mathrm{C}$ under a $14 \mathrm{~h}$ light $/ 10 \mathrm{~h}$ dark cycle with free access to food and water. Nine mice were randomly subdivided into three groups: vehicle group was injected with normal U87-MG cells; negative control group was injected with U87-MG cells transfected with control virus; LAMP-2A shRNA group was injected with U87-MG cells transfected with LMAP-2A shRNA virus. The allocation process was blinded to the researcher. Mice were injected subcutaneously under the axillary with treated U87-MG cells at a density of $1 \times 10^{7}$ and the tumor volume $\left(\mathrm{mm}^{3}=\right.$ length $\times$ width $\times$ height $\left.\times 0.5236\right)$ were calculated from day 12 until day 30 . All mice were sacrificed by intraperitoneal injection of $100 \mathrm{mg} / \mathrm{kg}$ of barbiturate and the tumor tissues were harvested as a whole to obtain weight before being cryopreserved for further analysis.

\section{Quantitative real-time RT-PCR}

Total RNA from cultured cells was extracted using TRIzol reagent (Invitrogen) according to the manufacturer's instructions. cDNA was generated by $1 \mu \mathrm{g}$ RNA using the RevertAid First Strand cDNA Synthesis Kit (MBI Fermentas, Burlington, Canada).

Quantitative real-time RT-PCR (qRT-PCR) was carried out with the Power SYBR Green Master Mix (Thermo Fisher Scientific, Wilmington, DE, USA) on ABI 7300 real-time quantitative PCR system (Life Technologies, Grand Island, NY) with the following conditions: $95{ }^{\circ} \mathrm{C}$ for $10 \mathrm{~min}, 40$ cycles of $95^{\circ} \mathrm{C}$ for $15 \mathrm{~s}, 60^{\circ} \mathrm{C}$ for $1 \mathrm{~min}$. The specific primers were list as follows: LAMP-2A, forward 5'CAATAGCAGCACCATTAAG3', reverse 5'GGAGCC ATTAACCAAATAC3; N-CoR, forward 5'AGGCGA CACAATCTTGAC $3^{\prime}$, reverse $5^{\prime}$ TGGAAGCGACAC TTTCAC $3^{\prime} ; \beta$-actin as internal reference, forward $5^{\prime}$ CATCGTCCACCGCAAATGCTTC $3^{\prime}$, reverse $5^{\prime}$ AAC CGACTGCTGTCACCTTCAC $3^{\prime}$. Results were analyzed using the $2^{-\Delta \Delta C}$ method.

\section{Western blot}

After various treatment, brain tissues or cell lysates were prepared with immunoprecipitation assay (RIPA) lysis buffer (solarbio, Dalian, China). BCA kit (Thermo Fisher Scientific, Wilmington, DE, USA) was applied to measure the protein concentrations. The lysates containing $40 \mu \mathrm{g}$ of protein were subjected to SDS-PAGE and transferred to nitrocellulose filter membrane (Millipore, Bedford, MA, USA). After blocking with 10\% bovine serum albumin at room temperature for $1 \mathrm{~h}$, the membranes were incubated overnight at $4{ }^{\circ} \mathrm{C}$ with primary antibodies as follows: LAMP-2A(1:1000, Abcam, Cambridge, UK, ab125068), N-CoR (1:500, Abcam, a ab58396), p-PERK (1:300, Santa Cruz, CA, USA, discontinued product, sc-32577) (1:1000, Cell Signaling Technology, Beverly, MA, \#3179S), PERK (1:1000, CST, \#5683S), $p$ IRE1 (1:2000, Abcam, ab124945), IRE1 (1:2000, Abcam, ab37073), $p$-JNK (1:1000, Abcam, ab124956), JNK (1:1000, Abcam, ab179461), CHOP (1:1000, Abcam, ab11419), Bcl-2 (1:300, Santa Cruz,sc-492), Bax (1:300, Santa Cruz, sc-493), Caspase-3(1:500, Abcam, ab44976), Caspase-9 (1:1000, Abcam, ab2013), GAPDH (1:2000, CST, \#5174), Hsc70(1:3000, Proteintech, IL, USA, 66,442-1-Ig), Lamin B1(1:1000, Abcam, ab16048) and $\beta$-actin(1:1000, CST, \#4970). Then the membranes were probed with horseradish peroxidase labeled secondary antibody (goat anti-rabbit, donkey anti-goat, goat antimouse, 1:1000, Beyotime, Shanghai, China), and visualized with chemiluminescence). NE-PER ${ }^{\mathrm{TM}}$ kit (Thermo Fisher Scientific) was used for nuclear and cytoplasmic protein extraction. The bands were quantified by Image software (ImageJ) from National Institutes of Health (http://rsb.info.nih.gov/ij/download.html).

\section{Immunohistochemistry staining}

Immunohistochemistry was performed with LAMP2A (1:100, Abcam, ab18528), N-CoR (1:100, Santa Cruz, sc-1609) for cells and N-CoR (1:100, Novus, CO, US, NBP1-28,863) for tissues. After being equilibrated at $-20{ }^{\circ} \mathrm{C}$ for $15 \mathrm{~min}$, brain tumor tissues were sectioned at a range of $5-7 \mu \mathrm{m}$ and fixed with $4{ }^{\circ} \mathrm{C}$ pre-cold 
(See figure on next page.)

Fig. 1 Expressive analysis of $L A M P-2 A$ and N-COR in clinical samples. a mRNA levels of LAMP-2A and N-CoR were measured by qRT-PCR. b Protein levels of LAMP-2A and N-CoR were measured by western blot. LAMP-2A mRNA and protein levels were significantly increased in GBM center $(n=8)$ in comparison with peri-tumor edema zone (PTEZ, $n=8)(p<0.0001$ ), while increasing trend was observed as compared with low grade glioma $(\mathrm{LGG}, \mathrm{n}=8)$. The protein level of N-CoR, but not mRNA level was significantly decreased in GBM center as compared with PTEZ $(p<0.0001)$. Linear regression analysis incorporating data from LGG, GBM center and PTEZ revealed moderate negative correlation between protein expression of LAMP-2A and that of N-CoR ( $r=-0.6001, p=0.0019)$. c Immunohistochemistry (IHC) analysis of LAMP-2A and N-CoR (brown signal) in glioma clinical samples. Nucleus (blue signal) was stained with hematoxylin; D. immunofluorescence (IF) analysis of LAMP-2A (green signal) and N-CoR (red signal) in glioma clinical samples. DNA (blue signal) was stained with DAPI. Both IHC and IF studies displayed upregulation of LAMP-2A and downregulation of $\mathrm{N}-\mathrm{COR}$ in GBM centers. The data are mean \pm SEM from 8 tissue specimens as a group. mRNA or protein levels are expressed relative to LGG set as 1 . Significant changes are set as $p<0.05$ and represented by asterisk (One-Way ANOVA; Bonferroni's test)

acetone. Cultured cells growing on glass slides were fixed with $4 \%$ of formaldehyde. The tissue or cell sections were incubated with $3 \%$ of $\mathrm{H}_{2} \mathrm{O}_{2}$ for 10 min, followed by $10 \%$ of BSA as blocking solution for $1 \mathrm{~h}$ at room temperature. Then the sections were incubated with the primary antibodies overnight at $4{ }^{\circ} \mathrm{C}$ and hybridized with horseradish peroxidase labeled secondary antibody (Beyotime, China) for $1 \mathrm{~h}$ at room temperature. The diaminobenzidine kit (DAB kit; Long Island Biotec, Shanghai, China) was applied for visualization and hematoxylin (BASO, China) for counterstain.

\section{Immunofluorescence staining}

The brain tissue and cell sections were prepared. After permeabilization with $0.3 \%$ Triton X-100 (SigmaAldrich), sections were probed with LAMP-2A (1:100, Abcam, ab18528), N-CoR (1:100, Novus, NBP1-28863) for tissues or N-CoR (1:100, Santa Cruz, sc-1609) for cells. After being washed with PBS containing $0.05 \%$ of Tween-20 (Sigma-Aldrich), sections were incubated with fluorescent secondary antibodies (Beyotime, China) and antifade mounting medium containing 1:500 DAPI (Beyotime, China). Leica fluorescent microscope was used for visualization.

\section{Co-immunoprecipitation (Co-IP)}

Cultured U87-MG cells were lysed in lysis buffer $(20 \mathrm{mM}$ pH 7.5 Tris, $150 \mathrm{mM} \mathrm{NaCl}$, 1\% TritonX-100, 1 mM EDTA and protease inhibitor cocktail). The lysates were cleared by centrifugation and subsequently incubated with either $20 \mu \mathrm{g}$ of IgG or $2 \mu \mathrm{g}$ of LAMP-2A antibody(Abcam, 125068), or $2 \mu \mathrm{g}$ of Hsc70 antibody(Proteintech, 10654-1AP) for $2 \mathrm{~h}$ with rotation on ice. Protein G-Agarose beads (Roche, Mannheim, Germany) were then added followed by overnight rotation at $4{ }^{\circ} \mathrm{C}$. Immunoprecipitants were separated on SDS-PAGE gel. LAMP-2A(1:1000, Abcam, 125068), Hsc70 (1:3000, Proteintech, 66442-1-Ig) and $\mathrm{N}-\mathrm{CoR}$ (1:500, Abcam, ab3482) were detected by western blotting as previously mentioned.

\section{Terminal deoxynucleotidyl transfer-mediated dUTP nick} end labeling (TUNEL)

Cultured cells growing on glass slides were fixed with $4 \%$ of formaldehyde. After permeabilization with $0.3 \%$ Triton X-100 (Sigma-Aldrich), apoptotic cells were detected according to the manufacturer's protocol of TUNEL kit (Roche).

\section{Flow cytometry detection of apoptosis}

Cultured cells growing on 6-well plate with various treatment were digested. Apoptosis was measured by the Annexin V-fluorescein isothiocyanate apoptosis kit (Beyotime, China) according to the manufacturer's protocol. The results were analyzed with a FACS Calibur flow cytometer (BD Biosciences).

\section{Statistical analysis}

Numerical data were expressed as means \pm standard error of mean (SEM) from at least 3 independent experiments. The statistical analyses were performed by OneWay ANOVA and Bonferroni's multiple comparisons test. All statistical analyses were performed with SPSS 18.0 software (SPSS, Inc., Chicago, IL), and $p<0.05$ was considered to be statistically significant.

\section{Results}

Expression of LAMP-2A in different regions of GBM and LGG

To determine the expressive profile of LAMP-2A in clinical samples, we analyzed mRNA by qRT-PCR, protein by western blot (WB), in-situ tissue expression by immunohistochemistry (IHC) and immunofluorescence (IF) in GBM center, PTEZ of GBM and LGG. Increased trend and significant increased expression of LAMP-2A at both mRNA and protein level were observed in GBM center compared with LGG and PTEZ relatively (Fig. 1a, b). Both IHC and IF analysis displayed upregulation of LAMP-2A in GBM center (Fig. 1c, d). The elevated expression of LAMP2A suggested the hypothesis that CMA might be playing a role in tumor formation. 


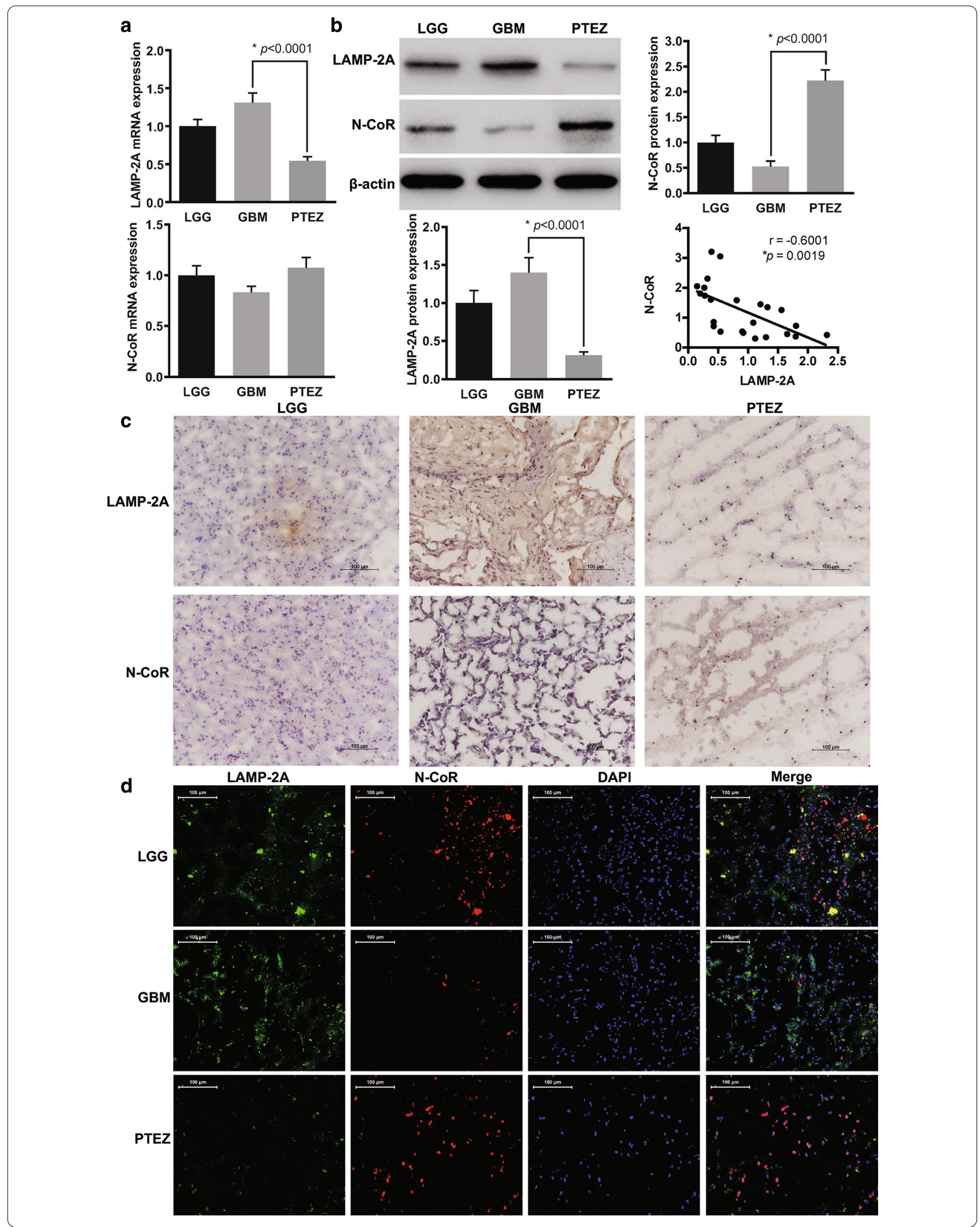




\section{(See figure on next page.)}

Fig. 2 Analysis of activation of UPR and caspase pathway and UPR in GBM samples with different LAMP-2A expression. a We divided six GBM samples according to the LAMP-2A level as "High" expression group and "Low" expression group. The average LAMP-2A expression of the High group was about two times of that of the Low group. $\mathbf{b}$ UPR activation was examined by protein expression of $p$-PERK, $p$-IRE1, $p$-JNK and CHOP. Significant down-regulation of $p$-PERK, $p$-IRE1, $p$-JNK and CHOP was observed in the High group as compared with the Low group. c Activator proteins in apoptosis including caspase 3, caspase 9 and Bax, and anti-apoptotic protein Bcl-2 were measured by western blot. Caspase 3, caspase 9 and Bax expressions were inhibited while BCl-2 expression was enhanced in the High group as compared with the Low group. $\mathbf{d}$ Apoptotic levels were examined by Tunnel analysis and compared within the two groups, with positive cells stained by orange. Patients with high LAMP-2A expression showed as half apoptosis rate as those with low LAMP-2A expression. The data are mean \pm SEM from three glioblastoma samples.

Protein levels are expressed relative to Low group set as 1. Significant changes are set as $p<0.05$ and represented by asterisk (Two-tailed t test)

\section{Post-transcriptional loss of $\mathrm{N}-\mathrm{CoR}$ in GBM was correlated with LAMP-2A expression}

The protein level of N-CoR, but not mRNA level was markedly decreased in GBM center as compared with PTEZ, as indicated by WB and qRT-PCR (Fig. 1a, b), implying post-transcriptional modification. Further linear regression analysis from protein expression profile of LGG, tumor center and PTEZ revealed moderate negative correlation between expression of LAMP-2A and that of N-CoR at protein level( $\mathrm{r}=-0.6001, p=0.0019)$. Both IHC and IF analysis confirmed the inverse association between LAMP-2A and N-CoR (Fig. 1c, d). These findings prompted us to explore the interaction between LAMP-2A and N-CoR, and the downstream pathway.

\section{Activation of UPR and caspase pathway in GBM with different LAMP-2A levels}

GBMs were divided into two groups according to LAMP2A level for further analysis of UPR and caspase pathway activation. Three patients with low LAMP-2A protein level were allocated to the "Low" group, and the other three patients with high LAMP-2A protein level to the "High" group. The average LAMP-2A expression in the High group was about twice as much as that in the Low group (Fig. 2a). Significant down-regulation of key components of UPR pathway, namely $p$-PERK, $p$-IRE1, $p$-JNK and CHOP, was detected in the High group compared with Low group (Fig. 2b). The apoptotic level in the High group was significantly lower than that in the Low group, as indicated by down-regulation of caspase 3, caspase 9 and Bax (pro-apoptotic protein), and up-regulation of Bcl-2 (anti-apoptotic protein) (Fig. 2c). TUNEL analysis revealed about half apoptotic rate in the High group than that in the Low group (Fig. 2d). The above findings in clinical samples led to the hypothesis that UPR and apoptotic pathway might be the downstream effector of CMA.

\section{Effect of LAMP-2A knockdown on CMA substrate}

The basal level of LAMP-2A was high in U87-MG cells (Fig. 3b, c). To explore the downstream effect of
LAMP-2A inhibition, we constructed three target siRNAs, among which LAMP-2A siRNA1 showed highest suppressive efficiency on qRT-PCR $(13.2 \%, p<0.0001)$ and was selected for the following experiments (Fig. 3a). Further WB and IHC confirmed the knockdown effect of LAMP-2A siRNA in contrast with the control siRNA (CT-siRNA) (Fig. 3b, c). CMA can be monitored indirectly by investigating its targeted proteins such as glyceraldehyde-3-phosphate dehydrogenase (GAPDH), which contains a KFERQ motif and is typically not regulated by transcriptional mechanisms [23]. The expression of GAPDH was slightly but significantly increased in U87-MG cells when LAMP-2A was silenced (Fig. 3b).

\section{$\mathrm{N}-\mathrm{CoR}$ interacted with LAMP-2A and was degraded by CMA in GBM cells}

It has been reported in non-small cell lung cancer that $\mathrm{N}$-CoR contains QEIFR pentapeptide and is the target of CMA [20]. Therefore, to test the direct association between N-CoR and LAMP-2A in GBM, together with Hsc70, another important component of CMA process which is responsible for targeting KFERQ-like-motifbearing proteins, co-immunoprecipitation assay was performed. The cell extract was immunoprecipitated with anti-LAMP-2A and anti-Hsc70 respectively, and the interaction with either LAMP-2A and Hsc-70 was confirmed by WB with anti-N-CoR (Fig. 4a). Moreover, from indirect immunofluorescence assay, significant co-localization between LAMP-2A and N-CoR was observed in vehicle and CT-siRNA treated U87-MG cells (Fig. 4b). We further examined the effect of LAMP-2A ablation on the expression and distribution of N-CoR. Treatment with LAMP-2A siRNA reversed $\mathrm{N}$-CoR loss at protein level, but not mRNA level (Fig. 4d, e). Upon activation of CMA by starvation, LAMP-2A expression dramatically increased, while $\mathrm{N}-\mathrm{CoR}$ expression decreased accordingly, as indicated by WB analysis (Fig. 4f). In immunofluorescence assay (Fig. 4b), upon siRNA mediated knockdown of cytoplasmic LAMP-2A, CMA mediated N-CoR ablation in 


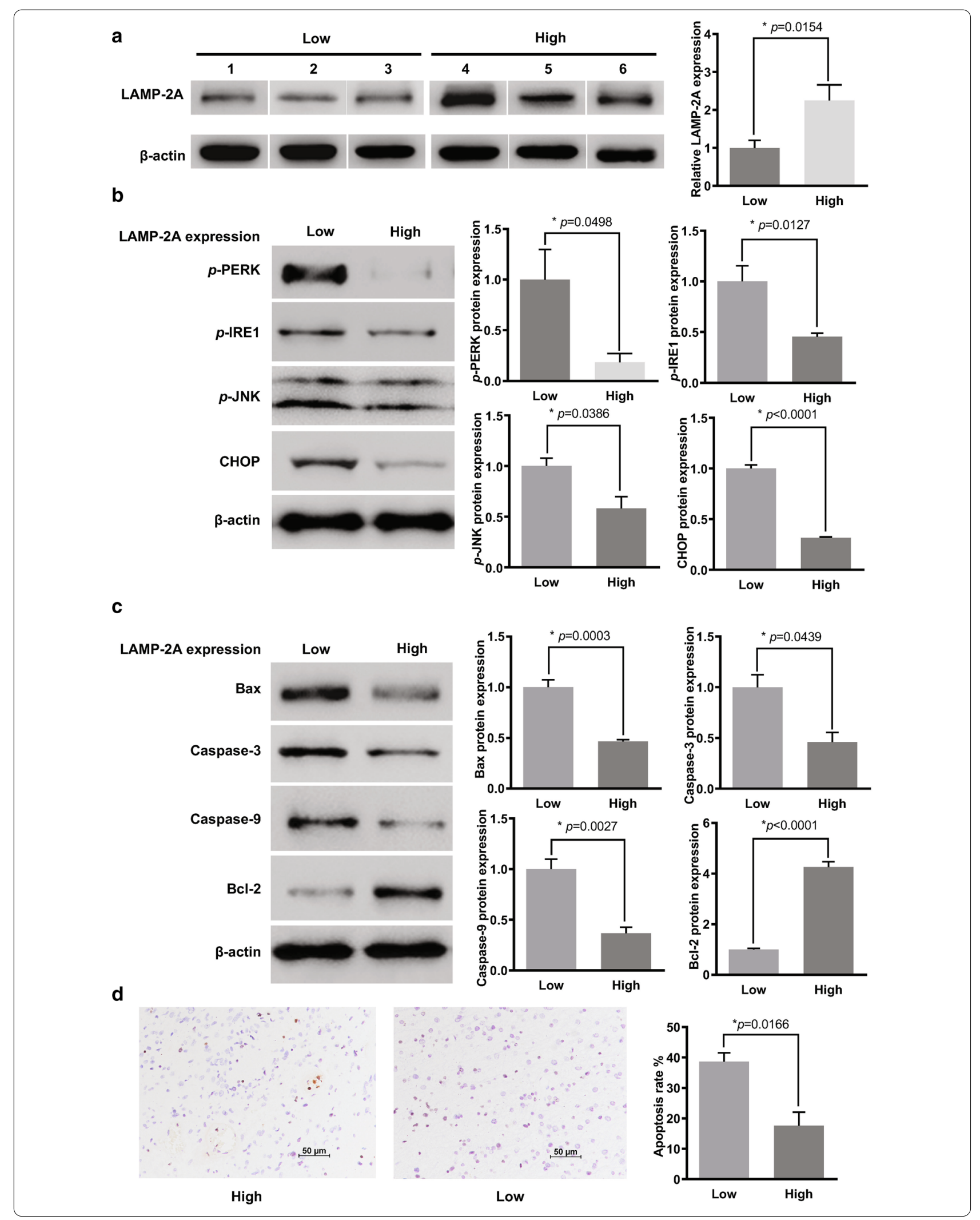




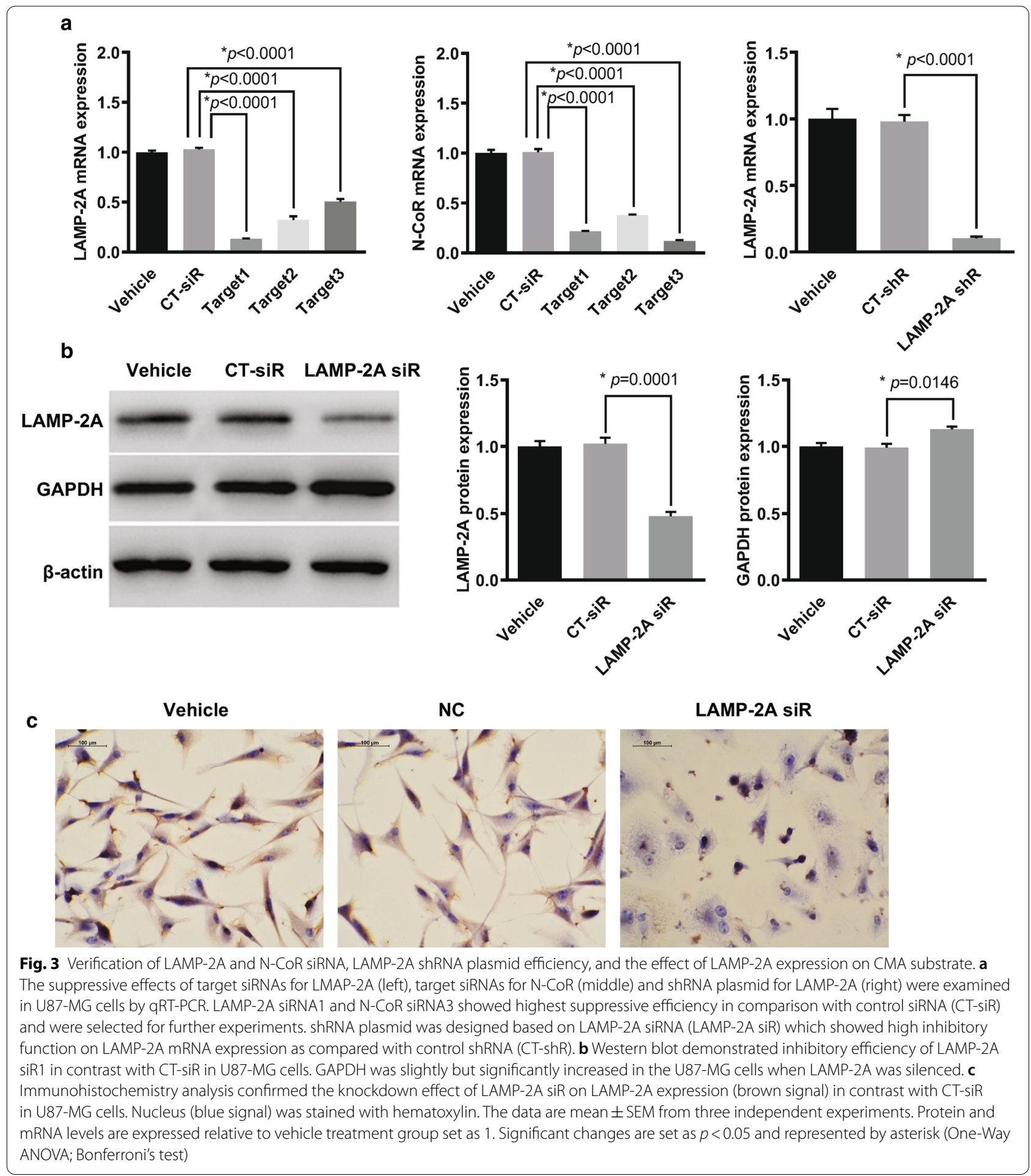

cytoplasm compartment was inhibited, and as a result remarkable cytoplasmic and intra-nucleus accumulation of N-CoR were observed. Since the remaining LAMP-2A also resided in the cytoplasm, it seemed that colocalization of both proteins increased. Furthermore, subcellular fractionation was conducted to separate nuclear and cytoplasmic proteins in CTsiRNA and LAMP-2A siRNA transfected cells, WB 


\begin{abstract}
(See figure on next page.)
Fig. $4 \mathrm{~N}$-CoR was a substrate of chaperone mediated autophagy and modulated by LAMP-2A siRNA. a The association between N-CoR and LAMP-2A (left), N-CoR and Hsc70 (right) was confirmed by co-immunoprecipitation assay in U87-MG cells. The cell lysates immunoprecipitated by either LAMP-2A antibody or Hsc70 displayed positive N-CoR protein expression as detected by N-CoR antibody. b Distribution of LAMP-2A (green) and N-CoR (red) in U87-MG cells was demonstrated in immunofluorescence analysis. DNA (blue signal) was stained with DAPI. Significant co-localization between LAMP-2A and N-CoR was observed in vehicle and control siRNA (CT-siR) groups. After treatment with LAMP-2A siRNA (LAMP-2A siR), remarkable cytoplasmic and intra-nucleus accumulation of $\mathrm{N}$-CoR was observed as compared with vehicle and CT-siR groups. c Nuclear and cytoplasmic proteins were separated by NE-PER ${ }^{\mathrm{TM}}$ kit. WB analysis showed that cytoplasmic LAMP-2A expression was inhibited, and $\mathrm{N}$-CoR in both cytoplasmic and nuclear compartments were accumulated upon treatment with LAMP-2A siRNA as compared with CT-siR treatment. $\mathbf{d}$ the protein expression of N-CoR was significantly increased after LAMP-2A knockdown as compared with CT-siR in U87-MG cells, while the mRNA level of $\mathrm{N}$-CoR remained unchanged. e Immunohistochemistry analysis confirmed increased protein level of N-CoR (brown signal) after LAMP-2A was inhibited in U87-MG cells. Nucleus (blue signal) was stained with hematoxylin. f Compared with normal control (NC), starvation overnight resulted in activation of CMA pathway, as demonstrated by increased protein expression of LAMP-2A, and significantly decreased N-CoR level. The data are mean \pm SEM from three independent experiments. Protein and mRNA levels are expressed relative to vehicle treatment group set as 1. Significant changes are set as $p<0.05$ and represented by asterisk (One-Way ANOVA; Bonferroni's test)
\end{abstract}

analysis confirmed knockdown of cytoplasmic LAMP$2 \mathrm{~A}$ and $\mathrm{N}-\mathrm{CoR}$ accumulation in both cytoplasmic and nuclear compartments upon treatment with LAMP-2A siRNA (Fig. 4c). These results proved that $\mathrm{N}-\mathrm{CoR}$ was degraded by CMA through recognition by Hsc70 and interaction with LAMP-2A and explained the inverse relationship between LAMP-2A and N-CoR observed in clinical samples.

\section{LAMP-2A knockdown enhanced apoptosis in GBM cells}

First we proved the siRNA mediated extinction of LAMP-2A and N-CoR protein expression by western blot (Fig. 5a). The apoptosis rate of U87-MG cells with suppressed LAMP-2A was remarkably enhanced when compared with control transfections, as displayed by TUNEL analysis (Fig. 5b). Additionally, flow cytometry showed both early and late apoptosis levels were elevated when LAMP-2A was silenced (Fig. 5c). Moreover, the pretreatment with $\mathrm{ZvaD}$, a pan-caspase inhibitor, resulted in the reversal of the pro-apoptotic effect of LAMP-2A silencing (Fig. 5c). These results supported the important role of LAMP-2A and CMA in improving GBM cell survival and promoting tumorigenesis, and the involvement of caspase pathway and apoptotic death in this process.

\section{$\mathrm{N}$-CoR knockdown reversed the pro-apoptosis function of LAMP-2A siRNA}

To prove whether CMA protected GBM cell from apoptosis through degradation of $\mathrm{N}-\mathrm{CoR}$, we designed and selected $\mathrm{N}-\mathrm{CoR}$ targeted siRNA with highest suppressive efficiency (Fig. 3a). Upon N-CoR knockdown (Fig. 5a), the enhanced apoptosis resulting from LAMP-2A ablation was truncated by half, as demonstrated by TUNEL and flow cytometry (Fig. 5b, c). The results suggested that GBM might evade from apoptosis via CMA mediated $\mathrm{N}$-CoR clearance.

\section{CMA inhibition resulted in activation of UPR and caspase pathway in $\mathrm{N}$-CoR dependent manner}

To test whether the protective effect of CMA on GBM cells was linked to neutralization of UPR, a stress reaction caused by intracellular accumulation of $\mathrm{N}-\mathrm{CoR}$, we further examined PERK/CHOP and IRE1/JNK pathway, which have been proved to fire apoptosis cascade [16-18]. The silencing efficiency of LAMP-2A siRNA and $\mathrm{N}$-CoR siRNA in U87-MG cells, alone or in combination, was proved as in Fig. 5a. LAMP-2A ablation resulted in remarkably increased expression of $p$-PERK, $p$-IRE1, $p$ JNK and CHOP (Fig. 6a). We further investigated the key proteins in the apoptosis pathway, and found significant up-regulation of caspase 3, caspase 9 and Bax with simultaneous down-regulation of Bcl-2 upon LAMP-2A inhibition (Figaure 6b). Furthermore, we examined the influence of N-CoR expression on the activated UPR and caspase pathway triggered by LAMP-2A ablation, and found that N-CoR knockdown achieved opposite effect, presented as decreased expression of $p$-PERK, $p$-IRE1, $p$-JNK, CHOP and apoptogenic proteins, and up regulation of anti-apoptotic protein, either by comparing with CT-siRNA group or LAMP-2A siRNA group (Fig. 6a, b). These findings collectively suggested that degradation of $\mathrm{N}-\mathrm{CoR}$ by CMA might protect GBM cells against UPR resulting from excessive $\mathrm{N}-\mathrm{CoR}$ accumulation and eventual avoid the activation of caspase pathway.

\section{CMA inhibition stunted the growth of glioma by restoring UPR-mediated apoptosis}

To explore the effects of CMA on in vivo glioblastoma growth, we established a xenograft nude mouse model. U87-MG cells were transfected with LAMP-2A shRNA virus to inhibit CMA activation (Fig. 3a). All the experimental mice were at similar healthy status before allocation and no procedure related adverse events 

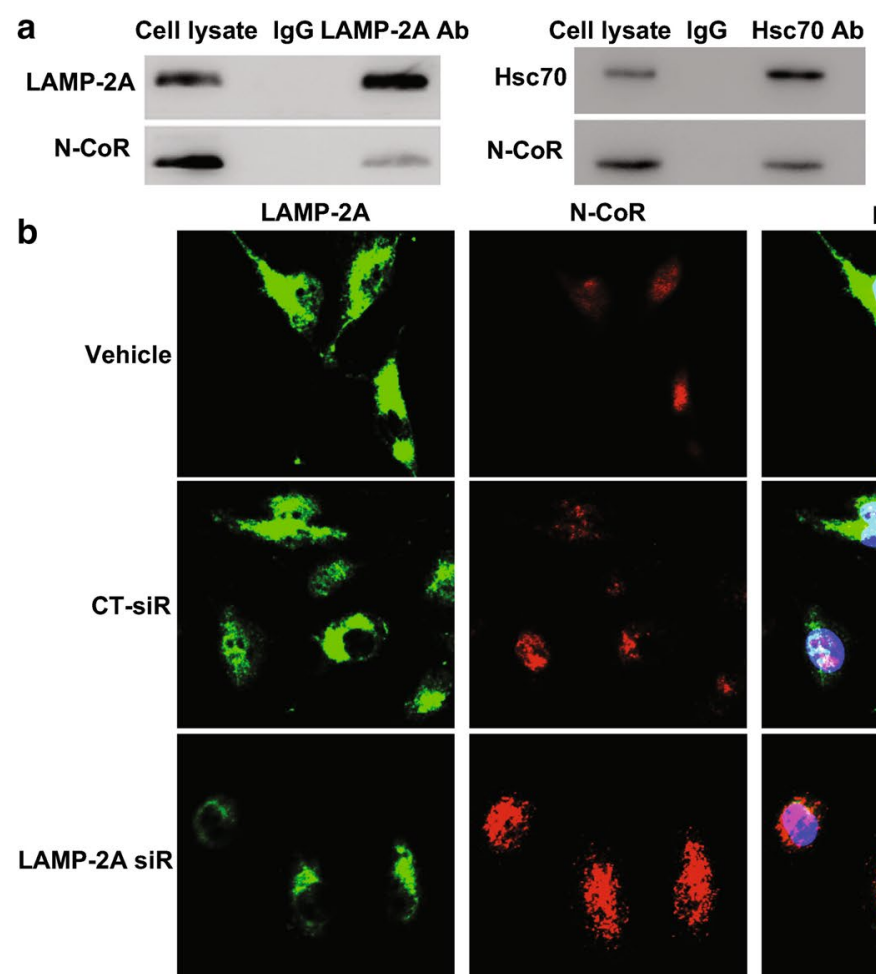

C
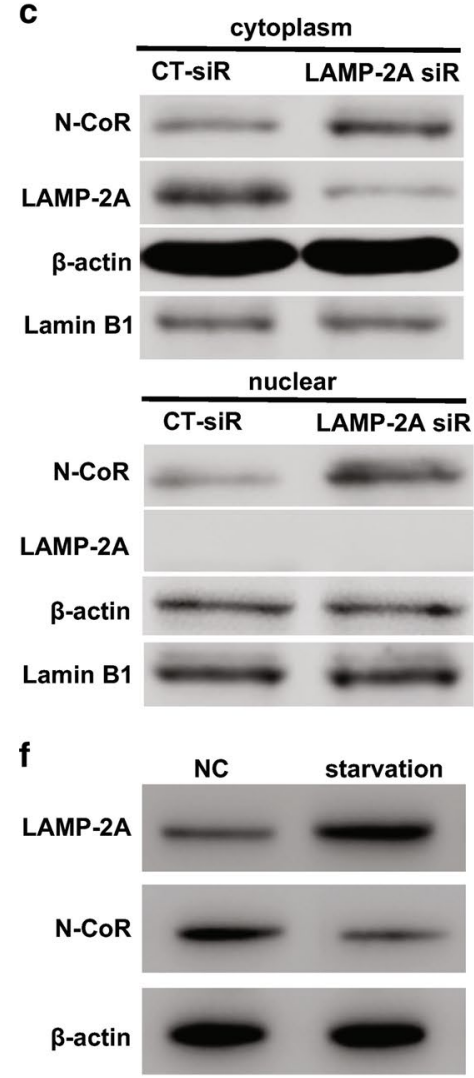

N-CoR
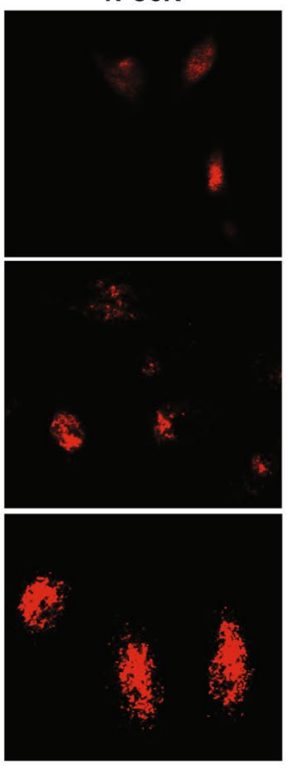

d
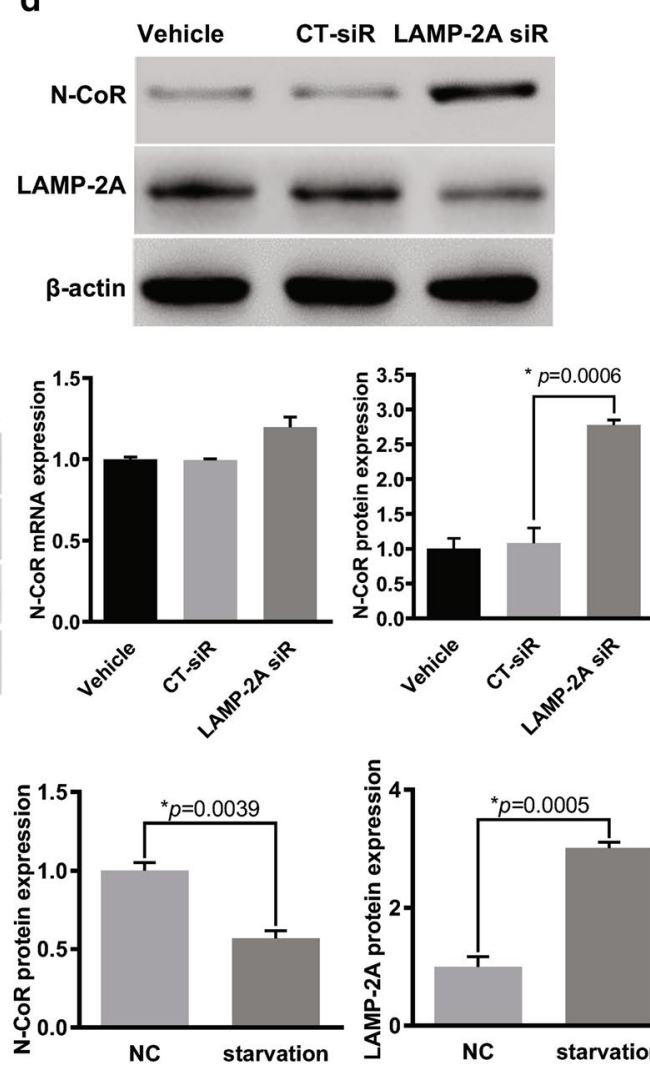

Merge
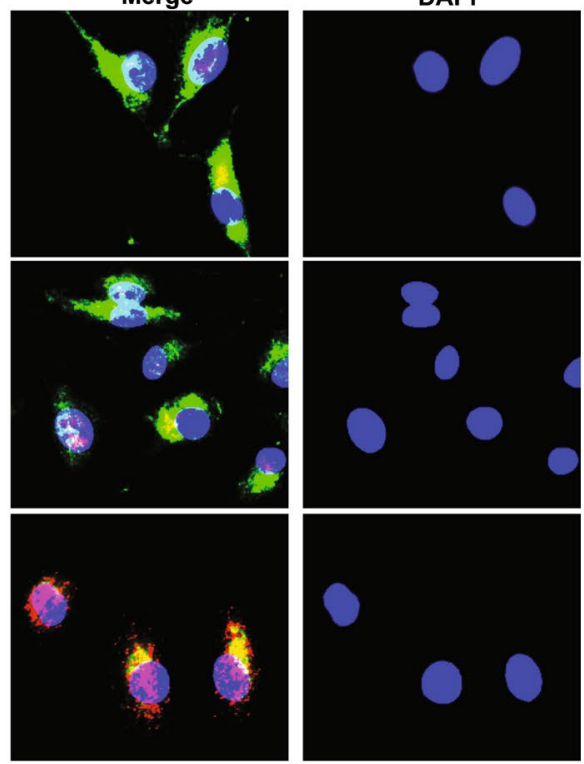

e

DAPI

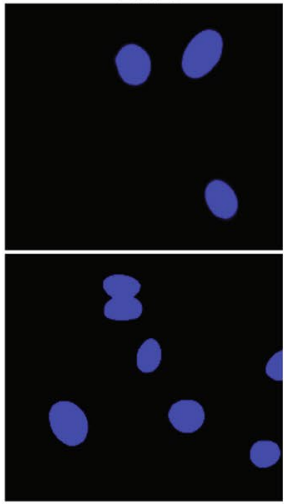

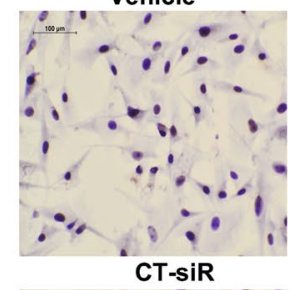
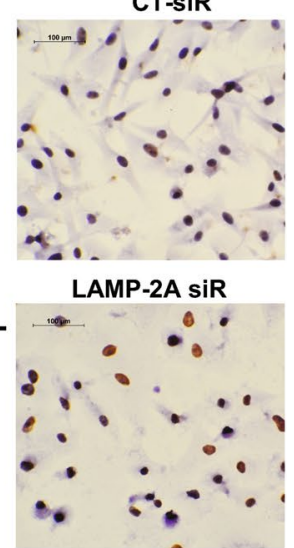

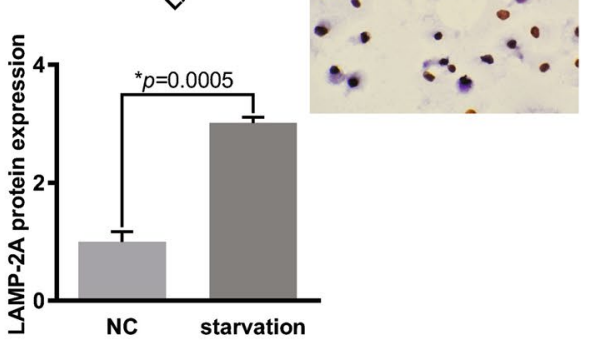




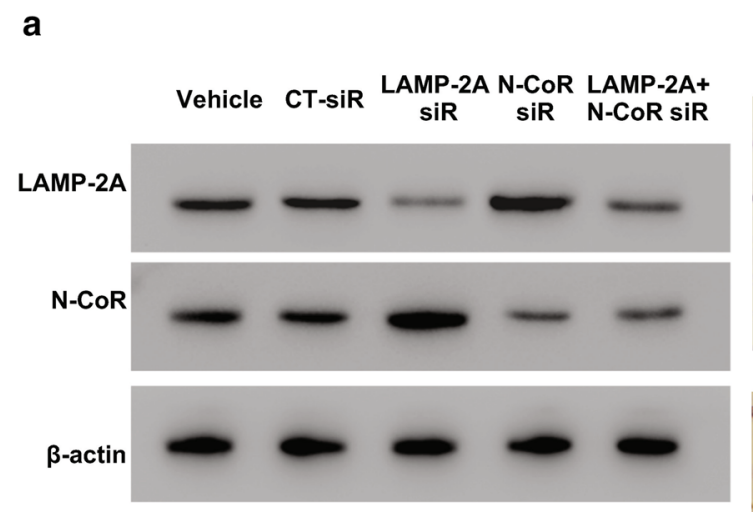

b
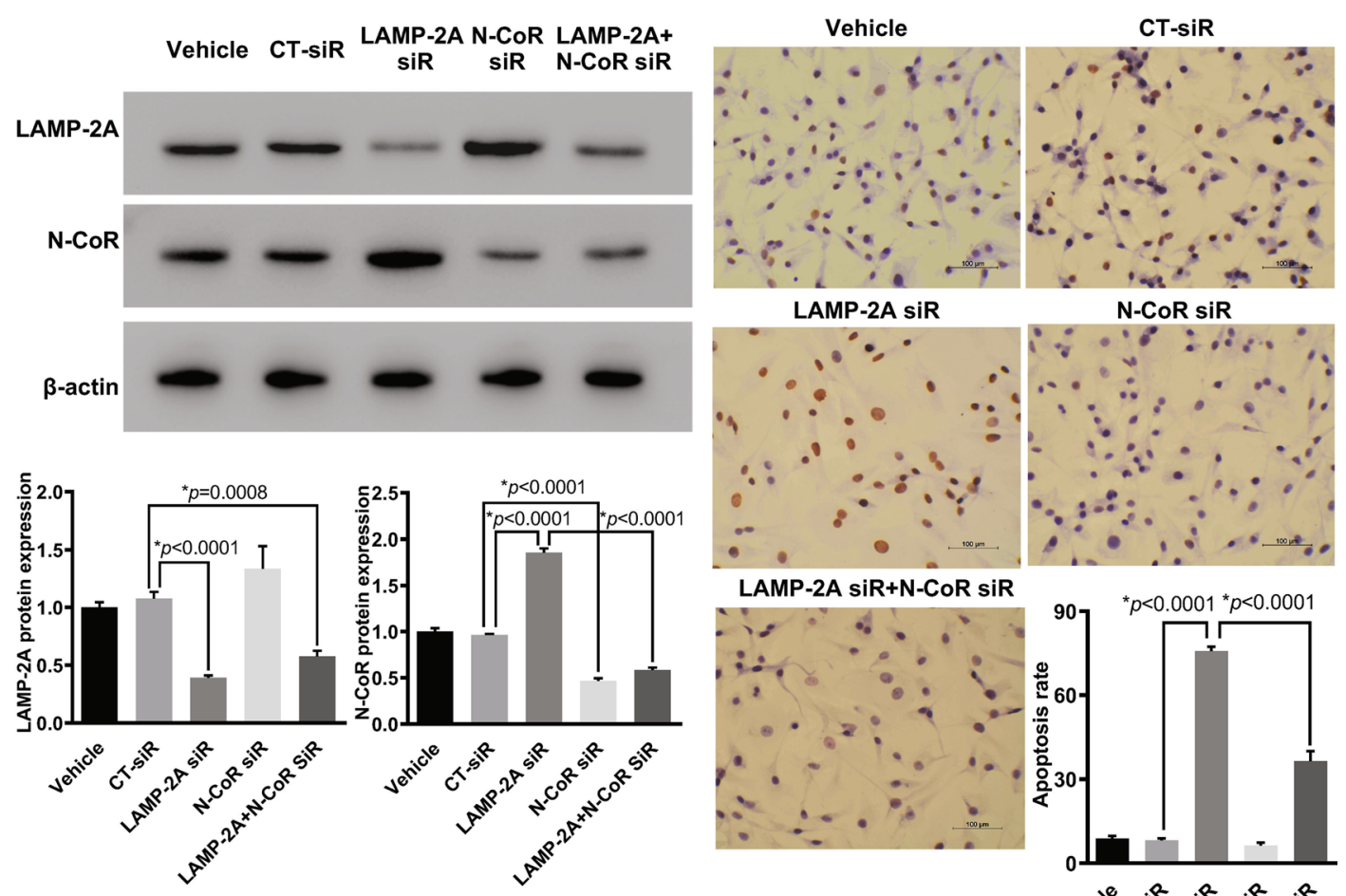

C
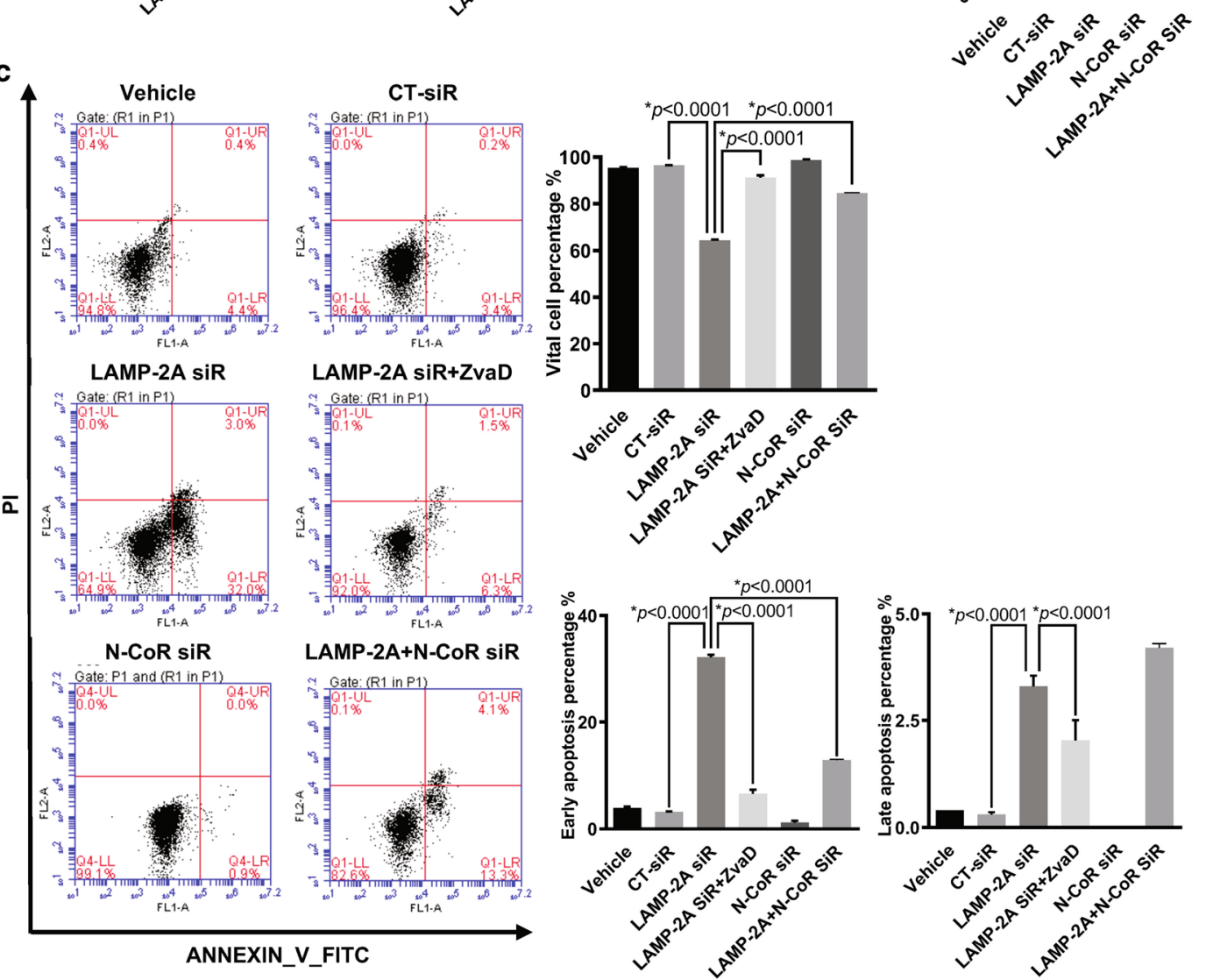


\section{(See figure on previous page.)}

Fig. 5 Effect of LAMP-2A and N-CoR expression on apoptosis in U87-MG cells. a Western blot analysis of LAMP-2A and N-CoR under different siRNA treatment conditions confirmed inhibitory effect of LAMP-2A siRNA (LAMP-2A siR) and N-CoR siRNA (N-CoR siR). Although LAMP-2A inhibition resulted in significant increase of $\mathrm{N}-\mathrm{COR}$, addition of $\mathrm{N}-\mathrm{COR}$ siR almost eliminated this effect. $\mathbf{b}$ TUNEL results of differently treated U87-MG cells. LAMP-2A silence by siRNA significantly enhanced the apoptotic level when compared with control siRNA (CT-siR) transfections, while addition of $\mathrm{N}$-CoR siR truncated the pro-apoptotic effect of LAMP-2A silence by half. c Flow cytometry analysis of differently treated U87-MG cells. Both early (right lower quadrant) and late apoptosis (right upper quadrant) levels were elevated when LAMP-2A was downregulated by siRNA. Again N-CoR ablation by siRNA eliminated enhanced apoptosis levels of LAMP-2A siR group by more than half. Treatment with ZvaD as pan-caspase inhibitor also reversed the pro-apoptotic effect of LAMP-2A ablation, confirming the involvement of caspase pathway as downstream effectors. The data are mean \pm SEM from three independent experiments. Significant changes are set as $p<0.05$ and represented by asterisk (One-Way ANOVA; Bonferroni's test)

were observed. Mice injected with LAMP-2A silenced U87-MG cells showed significantly smaller tumors than those in control shRNA (CT-shRNA) group (Fig. 7a). From post-implantation Day 18, the tumor volume was significantly smaller in mice from LAMP-2A shRNA group than CT-shRNA group, so as the final tumor weight after mice sacrifice (Fig. 7b, c). WB analysis of the tumor tissue demonstrated the decreased LAMP-2A and increased N-CoR expression in LAMP-2A shRNA group. Further IHC study confirmed the resultant accumulation of N-CoR after LAMP-2A being knocked down (Fig. 7e). We further examined activation of UPR after LAMP-2A inhibition, as revealed by significantly enhanced $p$-PERK, $p$-IRE1, $p$-JNK and CHOP expression by WB (Fig. 8a). Moreover, up-regulation of pro-apoptotic proteins and down-regulation of anti-apoptotic proteins were found in mice injected with LAMP-2A deficient U87-MG cells than those in control, which accorded with more apoptotic cells revealed by TUNEL analysis (Fig. 8b, c).

\section{Discussion}

Chaperone-mediated autophagy (CMA) is the first studied autophagy process indicating that lysosome guiding degradation of intracellular proteins can be selective [6]. Since binding of substrates to LAMP-2A is the limiting step, the CMA activity largely depends on the level of LAMP-2A [12]. Enhanced CMA via elevated LAMP$2 \mathrm{~A}$ expression has been reported in various malignant solid tumors including lung, breast and liver cancers to protect against intracellular stress and promote cancer cell survival $[8,10,11,20,24]$. Specific proteins bearing KFERQ-like motif such as ESP8, TP53, N-CoR, hypoxiainducible factor (HIF-1 $\alpha$ ) and PED are the downstream targets and mediate the oncogenic effect of CMA [10, 20, 24-28]. Physiologically brain tissue displays low expression of LAMP-2A. Under circumstances of stress and hypoxia, LAMP-2A is significantly elevated, resulting in activated CMA and contributing to cell survival [29]. As for GBM, elevated LAMP-2A was observed in clinical samples [30]. However, the essential function of LAMP$2 \mathrm{~A}$, and the involvement of CMA and downstream molecular mechanism in this lethal brain cancer, remain rarely investigated.

In this study, we found up-regulated LAMP-2A and inversely correlated $\mathrm{N}-\mathrm{CoR}$ expression at protein level in GBM patients. We further discovered suppressed UPR and apoptosis pathway in GBMs with high LAMP$2 \mathrm{~A}$ level. In vitro study with manipulated LAMP-2A and N-CoR expression demonstrated that CMA mediated N-CoR degradation and relieved UPR and apoptosis. In vivo xenograft model further proved that LAMP-2A silencing inhibited tumor growth and promoted the apoptosis. To the best of our knowledge, this study is the first work to provide evidence of LAMP-2A as potential biomarker and unveil the molecular mechanism of CMA mediated anti-apoptosis effect in GBM. Inhibiting CMA activity by targeting LAMP-2A can be exploited as a potential therapeutic approach in the treatment of malignant glioma.

\footnotetext{
(See figure on next page.)

Fig. 6 Effect of LAMP-2A and N-CoR expression on UPR activation and the apoptosis pathway in U87-MG cells. a UPR activation was examined by protein expression of $p$-PERK, $p$-IRE1, p-JNK and CHOP. LAMP-2A ablation resulted in remarkably increased expression of $p$-PERK, $p$-IRE1, $p$-JNK and CHOP, while N-CoR knockdown achieved opposite effect. These data together with findings from clinical samples, implied that CMA activation played important role in glioblastoma survival by degrading N-CoR and inhibiting downstream UPR activation. b Activator proteins in apoptosis including caspase 3, caspase 9 and Bax, and anti-apoptotic protein Bcl-2 were measured by western blot in differently treated U87-MG cells. Significant up-regulation of caspase 3, caspase 9 and Bax with simultaneous down-regulation of Bcl-2 were observed when LAMP-2A was inhibited, and these pro-apoptotic effects were reversed by $\mathrm{N}-\mathrm{CoR}$ knockdown. The data are mean $\pm \mathrm{SEM}$ from three independent experiments. The levels of $p$-PERK, $p$-IRE1 and $p$-JNK are compared with levels of PERK, IRE1 and JNK respectively, while the rest of the proteins are compared with $\beta$-actin. Protein levels are expressed relative to vehicle treatment group set as 1. Significant changes are set as $p<0.05$ and represented by asterisk (One-Way ANOVA; Bonferroni's test)
} 

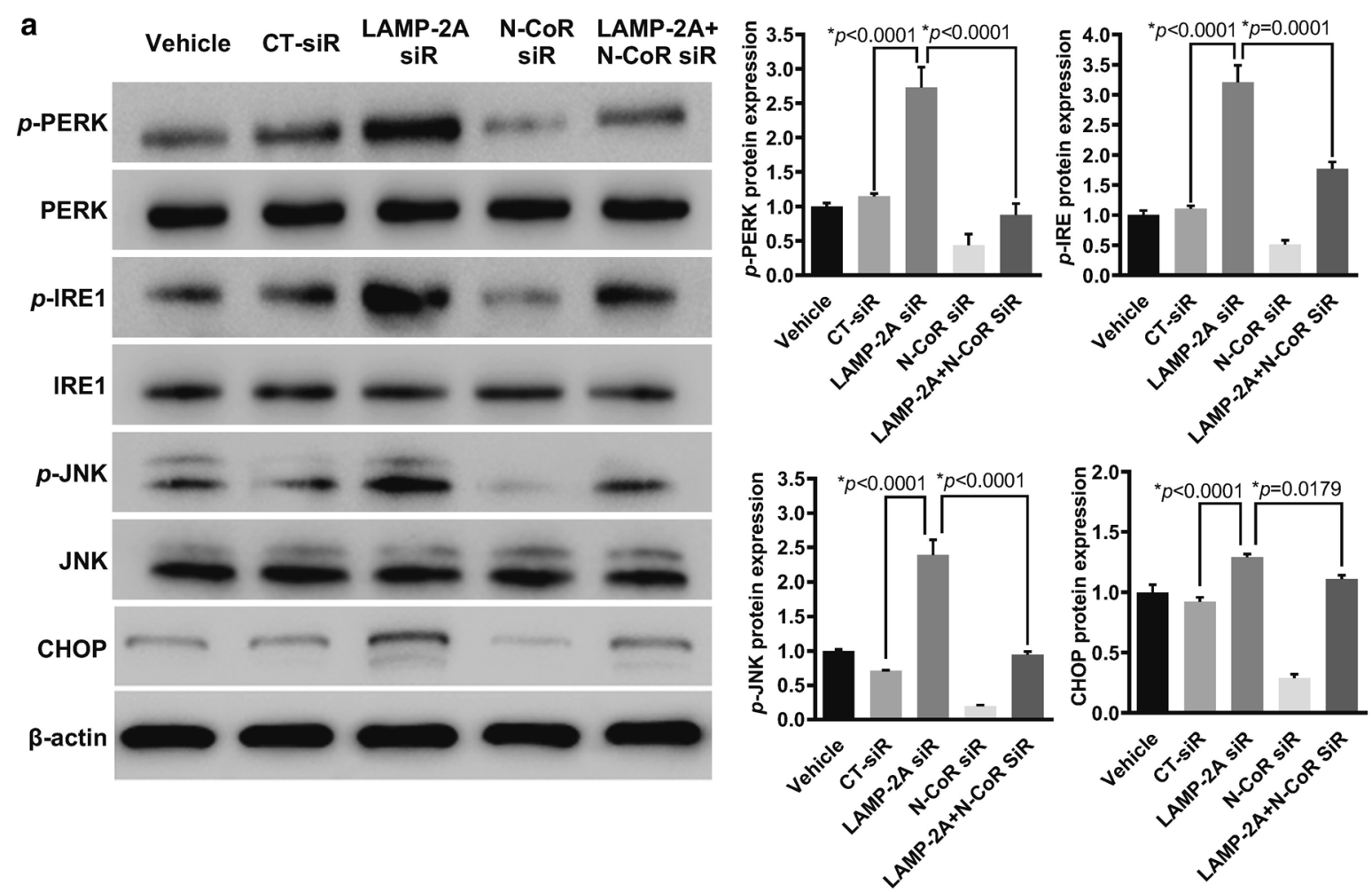

b
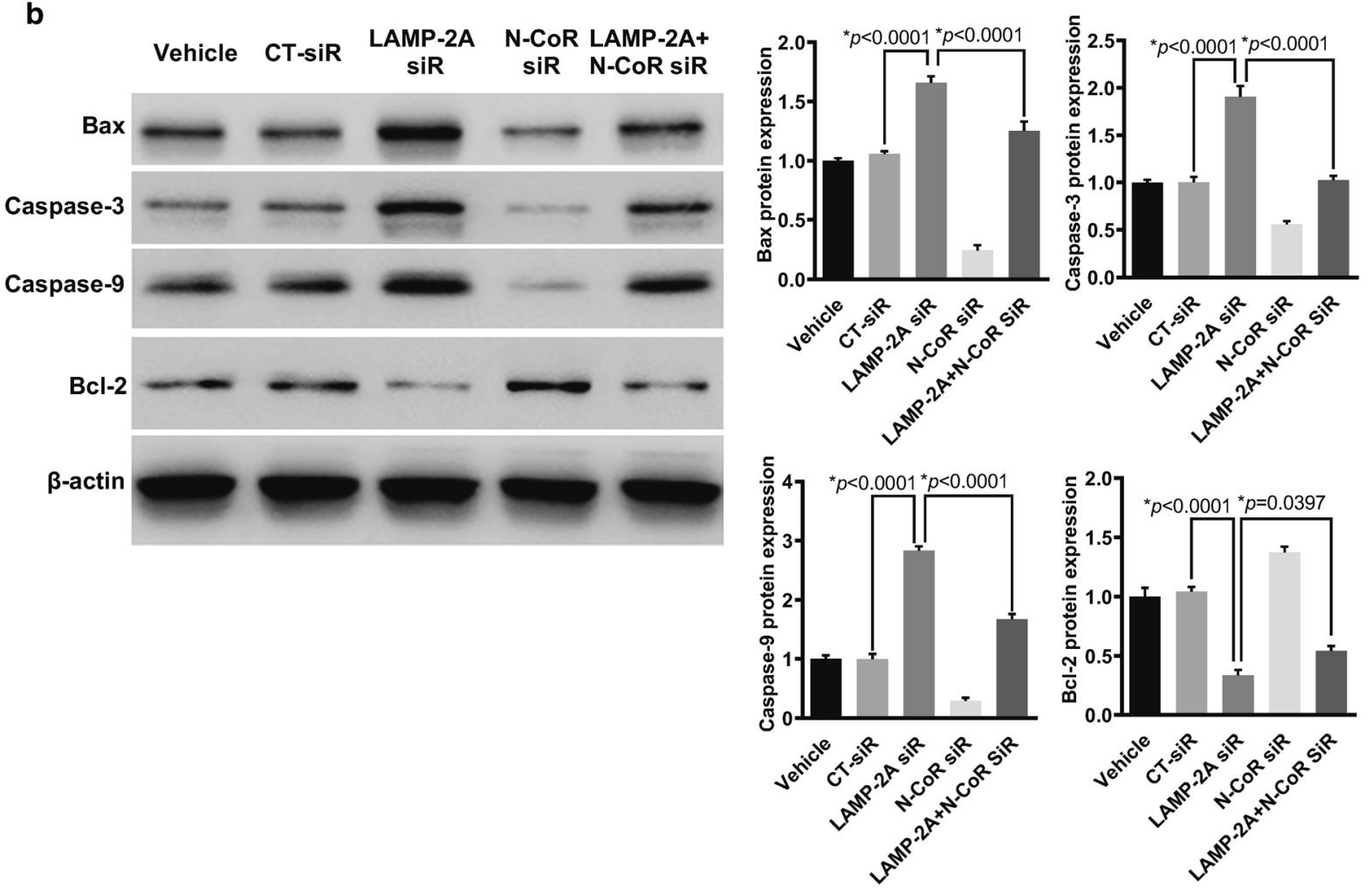


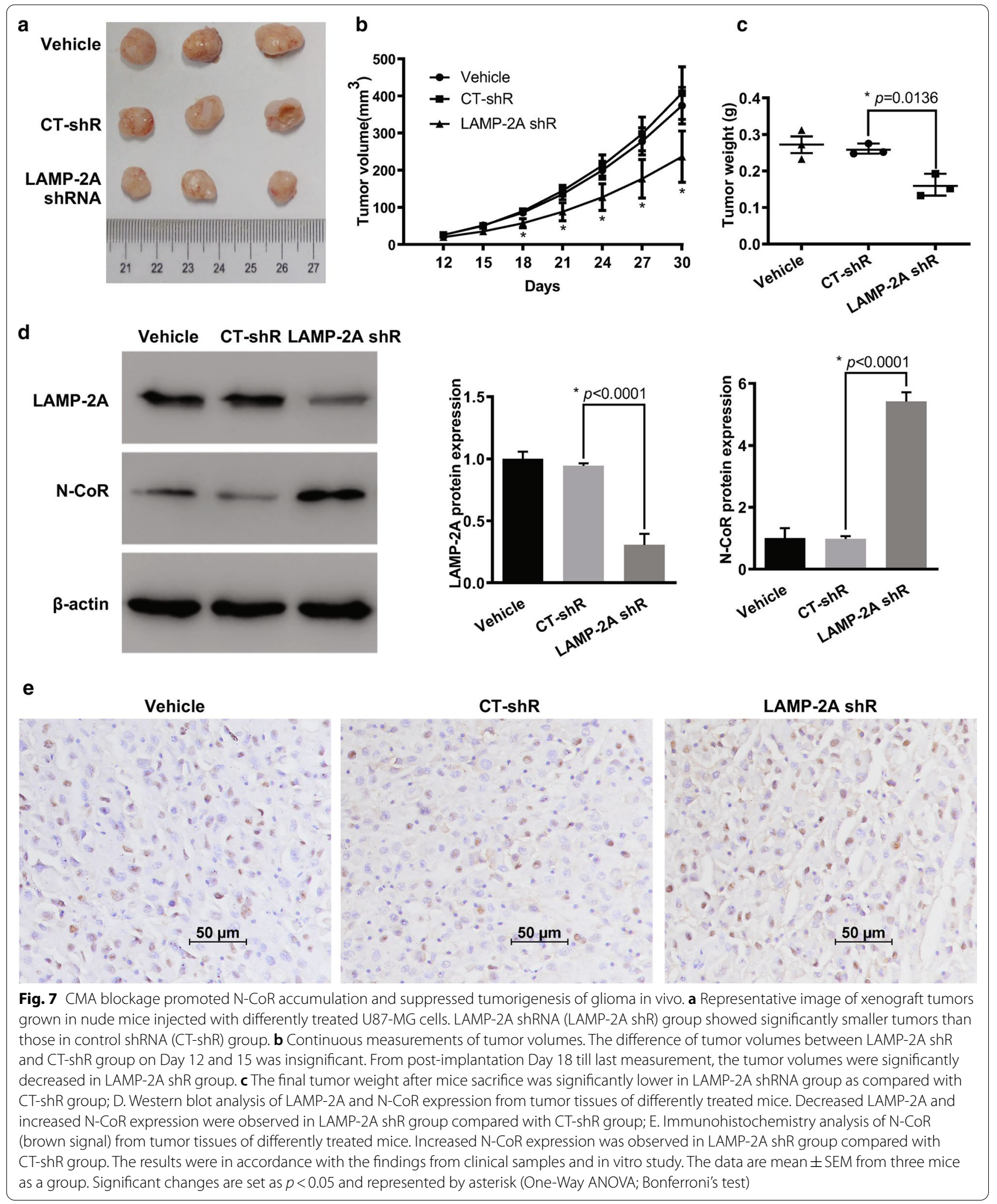


(See figure on next page.)

Fig. 8 Effect of CMA blockage on downstream UPR and apoptosis pathway in a xenograft mouse model. a UPR activation was examined by protein expression of $p$-PERK, $p$-IRE1, $p$-JNK and CHOP. Significant up-regulation of $p$-PERK, $p$-IRE1, $p$-JNK and CHOP was observed in the LAMP-2A shRNA (LAMP-2A shR) group as compared with control shRNA (CT-shR) group. b Activator proteins in apoptosis including caspase 3, caspase 9 and Bax, and anti-apoptotic protein BCl-2 were measured by western blot. Caspase 3, caspase 9 and Bax expressions were enhanced while Bcl-2 expression was inhibited in LAMP-2A shR group as compared with CT-shR group. c TUNEL results of differently treated groups. Significantly more apoptotic cells were found in LAMP-2A shR group as compared with CT-shR group. The data are mean \pm SEM from three mice as a group. The levels of $p$-PERK, $p$-IRE1 and $p$-JNK are compared with levels of PERK, IRE1 and JNK respectively, while the rest of the proteins are compared with $\beta$-actin. Protein levels are expressed relative to vehicle group set as 1. Significant changes are set as $p<0.05$ and represented by asterisk (One-Way ANOVA; Bonferroni's test)

Recently published research data identified the contribution of GBM cells to the up-regulation of LAMP-2A and CMA activity in pericytes through cell-cell interaction, which ablated pericytes antitumor immune function essential for GBM survival [12]. However, in terms of temozolomide (TMZ) treatment, in vitro studies showed CMA mediated downregulation of HIF-1 $\alpha$ improved the response of TMZ-resistant GBM cells, and blocking CMA induced resistance of TMZ-sensitive cells [13, 14]. While in breast cancer inhibiting CMA by downregulating LAMP-2A could augment sensitivity to doxorubicin [11]. Therefore, the role of CMA in GBM tumorigenesis and chemotherapy is more than a unilateral modulation and in-depth research is worthwhile to unveil the complex tumor biology and facilitate the development of anti-GBM strategy.

We demonstrated that the anti-apoptotic effect of CMA in GBM was through degradation of N-CoR and abrogation of downstream UPR. As the core component of a multi-protein repressor complex, N-CoR regulates transcription of various tumor suppressor genes [31]. GBM cell with low or inhibited N-CoR expression showed enhanced invasiveness and increased tumor formation capacity $[21,22]$. On the other hand, serine/ threonine protein phosphatase 2A (PP2A) inhibitor or combination of retinoic acid and okadaic acid led to disruption of N-CoR complex and its cytoplasmic translocation, and resulted in tumor cell differentiation and death $[22,32,33]$. The phenomenon is attributed to the cytotoxic ER stress UPR induced by uncontrollable cytosolic accumulation of N-CoR [19, 34]. Cancer cells benefit from the loss of function of N-CoR complex and escape from the side effect of N-CoR translocation by means of elevated LAMP-2A and CMA mediated clearance [19, $20]$.
N-CoR/UPR does not fully account for the protective effect of CMA in GBM, since N-CoR elimination by siRNA could not completely reverse the activation of apoptosis resulted from LAMP-2A inhibition. As a matter of fact, CMA impacts multiple complex pathways to promote cancer survival. Tumor cells induce acetylation of PKM2 by high glucose concentration and deliver it to CMA degradation in order to accumulate glycolytic intermediates for cell growth [28]. CMA is also responsible for degradation of $\mathrm{p} 53$, which induces transcriptional downregulation of various glycolytic enzymes essential for tumor growth $[8,35]$. Elevating CMA activity by downregulating autophagy-related gene 5 (ATG5)-dependent macroautophagy stimulates growth and metastasis of breast cancer cells [36]. Similarly, induction of CMA compensates for impaired macroautophagy to promote hepatocellular carcinoma survival [37]. Considering the diverse pathways related to CMA in other cancer types, more researches are worthwhile to depict a complete regulatory network of CMA in GBM development.

\section{Conclusions}

In conclusion, our research proved that LAMP-2A upregulation protected GBM cells from apoptosis by degrading $\mathrm{N}-\mathrm{CoR}$ and inhibiting downstream UPR. The mechanism of CMA shift between pro-oncogenic effect and chemoresistance to TMZ still remains to be elucidated. Further insights into the interplay between CMA and other biological process essential for GBM development are needed. Last but not least, as a promising biomarker for GBM development, LAMP-2A has remarkable therapeutic implication and effective modulators remain to be investigated. 


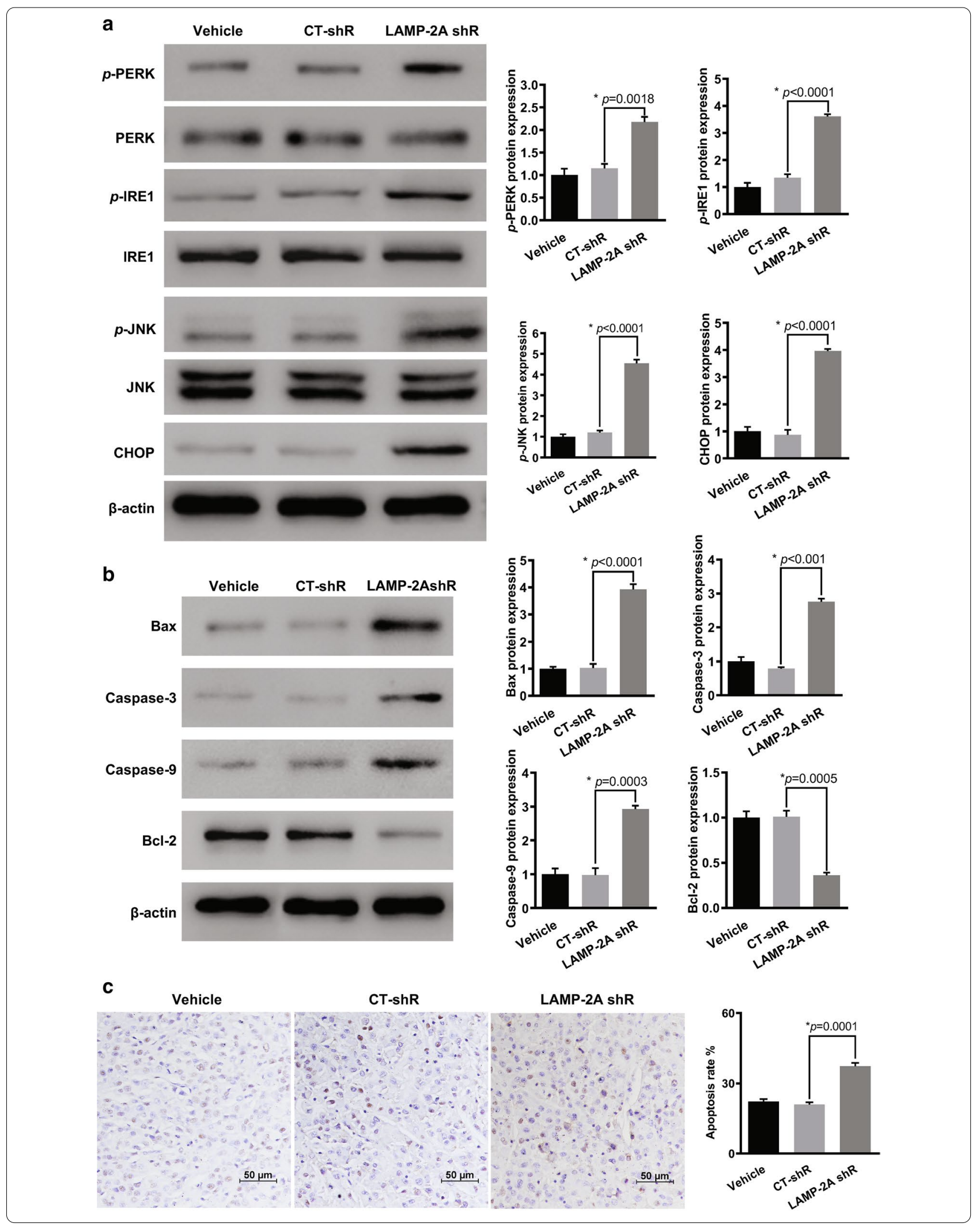




\section{Abbreviations}

GBM: GlioblastomaGBM; CMA: Chaperone-mediated autophagy; Hsc70: Heat shock cognate $71 \mathrm{kDa}$ protein; LAMP-2A: Lysosomal associated membrane protein 2A; N-CoR: Nuclear receptor co-repressor; UPR: Unfolded protein response; PERK: Pancreatic ER kinase; elF2a: Eukaryotic translation initiator factor 2a; ATF4: Activating transcription factor 4; CHOP: C/EBP homologous protein; IRE1: Inositol requiring enzyme 1; TRAF2: TNF receptor-associated factor 2; ASK1: Apoptosis signal regulating kinase 1; JNK: JUN N-terminal kinase; LGG: Low grade glioma; PTEZ: Peri-tumor edema zone; qPCR: Quantitative polymerase chain reaction; WB: Western blot; IHC: Immunohistochemistry; DMEM: Dulbecco's modified Eagle's medium; FBS: Fetal bovine serum; TUNEL: Terminal deoxynucleotidyl transfer-mediated dUTP nick end labeling; qRT-PCR: Quantitative real-time RT-PCR; HIF-1a: Hypoxia-inducible factor; PP2A: Serine/ threonine protein phosphatase 2A; ATG5: Autophagy-related gene 5; Co-IP: Co-immunoprecipitation; CT-siRNA: Control siRNA; CT-shRNA: Control shRNA.

\section{Acknowledgements}

The authors have no acknowledgement to disclose.

\section{Authors' contributions}

WYJ did substantial contributions to conception, design, conducting the in vitro experiments and drafting the article; ZBY collected the clinical samples and analyzed the clinical data; WJL conducted the in vivo experiments; WHJ and XSB built up all the molecular tools for the research; WJL; Conduct part of the experiments: WHJ and XSB; ZJM and WL supervised the whole research and critically revised the manuscript to the approval of the final version; $W L$ agreed to be accountable for all aspects of the work in ensuring that questions related to the accuracy or integrity of the work were appropriately investigated and resolved. All authors read and approved the final manuscript.

\section{Funding}

The whole work was supported by grants from the National Natural Science Foundation of China (81502139).

\section{Availability of data and materials}

The datasets used and/or analysed during the current study are available from the corresponding author on reasonable request.

\section{Declarations}

\section{Ethics approval and consent to participate}

Approval of the study was obtained from the Ethics Committee of the Second Affiliated Hospital of Zhejiang University, School of Medicine. The experimental protocol was established, according to the ethical guidelines of the Helsinki Declaration and was approved by the Human and Animal Ethics Committee of the Second Affiliated Hospital of Zhejiang University, School of Medicine. Written informed consent was obtained from individual or guardian participants.

\section{Consent for publication}

Informed written consent was obtained from the patients for publication of this manuscript and accompanying images.

\section{Competing interests}

The authors declare that they have no competing interests.

\section{Author details}

${ }^{1}$ Department of Neurosurgery, 2Nd Affiliated Hospital, School of Medicine, Zhejiang University, 88\# Jiefang Road, Hangzhou 310009, Zhejiang, China. ${ }^{2}$ Department of Pathology, 2Nd Affiliated Hospital, School of Medicine, Zhejiang University, 88\# Jiefang Road, Hangzhou 310009, Zhejiang, China.

Received: 4 October 2020 Accepted: 22 February 2021 Published online: 24 March 2021

\section{References}

1. Stupp R, Mason WP, van den Bent MJ, Weller M, Fisher B, Taphoorn MJ, et al. Radiotherapy plus concomitant and adjuvant temozolomide for glioblastoma. N Engl J Med. 2005;352(10):987-96.

2. Nam JY, de Groot JF. Treatment of Glioblastoma. J Oncol Pract. 2017;13(10):629-38.

3. Kaka N, Hafazalla K, Samawi H, Simpkin A, Perry J, Sahgal A, et al. Progression-free but no overall survival benefit for adult patients with bevacizumab therapy for the treatment of newly diagnosed glioblastoma: a systematic review and meta-analysis. Cancers (Basel). 2019;11(11):1723.

4. Rabinowitz JD, White E. Autophagy and metabolism. Science. 2010;330(6009):1344-8.

5. Catarino S, Pereira P, Girao H. Molecular control of chaperone-mediated autophagy. Essays Biochem. 2017;61(6):663-74.

6. Kaushik S, Cuervo AM. The coming of age of chaperone-mediated autophagy. Nat Rev Mol Cell Biol. 2018;19(6):365-81.

7. Wu H, Chen S, Ammar AB, Xu J, Wu Q, Pan K, et al. Crosstalk between macroautophagy and chaperone-mediated autophagy: implications for the treatment of neurological diseases. Mol Neurobiol. 2015:52(3):1284-96.

8. Kon M, Kiffin R, Koga H, Chapochnick J, Macian F, Varticovski L, et al. Chaperone-mediated autophagy is required for tumor growth. Sci Transl Med. 2011;3(109):109ra17.

9. Cuervo AM, Wong E. Chaperone-mediated autophagy: roles in disease and aging. Cell Res. 2014;24(1):92-104.

10. Thorburn A, Debnath J. Targeting chaperone-mediated autophagy in cancer. Sci Transl Med. 2011;3(109):109ps45.

11. Saha T. LAMP2A overexpression in breast tumors promotes cancer cell survival via chaperone-mediated autophagy. Autophagy. 2012;8(11):1643-56

12. Valdor R, Garcia-Bernal D, Riquelme D, Martinez CM, Moraleda JM, Cuervo $\mathrm{AM}$, et al. Glioblastoma ablates pericytes antitumor immune function through aberrant up-regulation of chaperone-mediated autophagy. Proc Natl Acad Sci U S A. 2019;116(41):20655-65.

13. Lo Dico A, Salvatore D, Martelli C, Ronchi D, Diceglie C, Lucignani G, et al. Intracellular redox-balance involvement in temozolomide resistancerelated molecular mechanisms in glioblastoma. Cells. 2019;8(11):1315

14. Lo Dico A, Martelli C, Diceglie C, Lucignani G, Ottobrini L. Hypoxia-inducible factor-1alpha activity as a switch for glioblastoma responsiveness to temozolomide. Front Oncol. 2018:8:249.

15. Horlein AJ, Naar AM, Heinzel T, Torchia J, Gloss B, Kurokawa R, et al. Ligand-independent repression by the thyroid hormone receptor mediated by a nuclear receptor co-repressor. Nature. 1995;377(6548):397-404.

16. Ng AP, Howe Fong J, Sijin Nin D, Hirpara JL, Asou N, Chen CS, et al. Cleavage of misfolded nuclear receptor corepressor confers resistance to unfolded protein response-induced apoptosis. Cancer Res. 2006:66(20):9903-12.

17. Hetz C, Chevet E, Harding HP. Targeting the unfolded protein response in disease. Nat Rev Drug Discov. 2013;12(9):703-19.

18. Vandewynckel YP, Laukens D, Geerts A, Bogaerts E, Paridaens A, Verhelst $X$, et al. The paradox of the unfolded protein response in cancer. Anticancer Res. 2013;33(11):4683-94.

19. Khan M. Interplay of protein misfolding pathway and unfolded-protein response in acute promyelocytic leukemia. Expert Rev Proteomics. 2010;7(4):591-600.

20. Ali AB, Nin DS, Tam J, Khan M. Role of chaperone mediated autophagy (CMA) in the degradation of misfolded N-CoR protein in non-small cell lung cancer (NSCLC) cells. PLoS ONE. 2011;6(9):e25268.

21. Verhaak RG, Hoadley KA, Purdom E, Wang V, Qi Y, Wilkerson MD, et al. Integrated genomic analysis identifies clinically relevant subtypes of glioblastoma characterized by abnormalities in PDGFRA, IDH1, EGFR, and NF1. Cancer Cell. 2010;17(1):98-110.

22. Heldring N, Nyman U, Lonnerberg P, Onnestam S, Herland A, Holmberg J, et al. NCoR controls glioblastoma tumor cell characteristics. Neuro Oncol. 2014; 16(2):241-9. 
23. Kiffin R, Christian C, Knecht E, Cuervo AM. Activation of chaperone-mediated autophagy during oxidative stress. Mol Biol Cell. 2004; 15(11):4829-40

24. Quintavalle C, Di Costanzo S, Zanca C, Tasset I, Fraldi A, Incoronato M, et al. Phosphorylation-regulated degradation of the tumor-suppressor form of PED by chaperone-mediated autophagy in lung cancer cells. Cell Physiol. 2014;229(10):1359-68.

25. Welsch T, Younsi A, Disanza A, Rodriguez JA, Cuervo AM, Scita G, et al. Eps 8 is recruited to lysosomes and subjected to chaperone-mediated autophagy in cancer cells. Exp Cell Res. 2010;316(12):1914-24.

26. Hubbi ME, Hu H, Ahmed I, Levchenko A, Semenza GL. Chaperone-mediated autophagy targets hypoxia-inducible factor-1alpha (HIF-1alpha) for lysosomal degradation. J Biol Chem. 2013;288(15):10703-14.

27. Vakifahmetoglu-Norberg H, Kim M, Xia HG, Iwanicki MP, Ofengeim D, Coloff JL, et al. Chaperone-mediated autophagy degrades mutant p53. Genes Dev. 2013;27(15):1718-30.

28. Lv L, Li D, Zhao D, Lin R, Chu Y, Zhang H, et al. Acetylation targets the M2 isoform of pyruvate kinase for degradation through chaperone-mediated autophagy and promotes tumor growth. Mol Cell. 2011;42(6):719-30.

29. Dohi E, Tanaka S, Seki T, Miyagi T, Hide I, Takahashi T, et al. Hypoxic stress activates chaperone-mediated autophagy and modulates neuronal cell survival. Neurochem Int. 2012;60(4):431-42.

30. Giatromanolaki A, Sivridis E, Mitrakas A, Kalamida D, Zois CE, Haider S, et al. Autophagy and lysosomal related protein expression patterns in human glioblastoma. Cancer Biol Ther. 2014;15(11):1468-78.

31. Battaglia S, Maguire $O$, Campbell MJ. Transcription factor co-repressors in cancer biology: roles and targeting. Int J Cancer. 2010;126(11):2511-9.
32. Park DM, Li J, Okamoto H, Akeju O, Kim SH, Lubensky I, et al. N-CoR pathway targeting induces glioblastoma derived cancer stem cell differentiation. Cell Cycle. 2007;6(4):467-70.

33. Lu J, Zhuang Z, Song DK, Mehta GU, Ikejiri B, Mushlin H, et al. The effect of a PP2A inhibitor on the nuclear receptor corepressor pathway in glioma. J Neurosurg. 2010;113(2):225-33.

34. Khan MM, Nomura T, Chiba T, Tanaka K, Yoshida H, Mori $\mathrm{K}$, et al. The fusion oncoprotein PML-RARalpha induces endoplasmic reticulum (ER)-associated degradation of N-CoR and ER stress. J Biol Chem. 2004;279(12):11814-24.

35. Jiang P, Du W, Wang X, Mancuso A, Gao X, Wu M, et al. p53 regulates biosynthesis through direct inactivation of glucose-6-phosphate dehydrogenase. Nat Cell Biol. 2011;13(3):310-6.

36. Han Q, Deng Y, Chen S, Chen R, Yang M, Zhang Z, et al. Downregulation of ATG5-dependent macroautophagy by chaperone-mediated autophagy promotes breast cancer cell metastasis. Sci Rep. 2017;7(1):4759.

37. Chava S, Lee C, Aydin Y, Chandra PK, Dash A, Chedid M, et al. Chaperonemediated autophagy compensates for impaired macroautophagy in the cirrhotic liver to promote hepatocellular carcinoma. Oncotarget. 2017;8(25):40019-36.

\section{Publisher's Note}

Springer Nature remains neutral with regard to jurisdictional claims in published maps and institutional affiliations.
Ready to submit your research? Choose BMC and benefit from:

- fast, convenient online submission

- thorough peer review by experienced researchers in your field

- rapid publication on acceptance

- support for research data, including large and complex data types

- gold Open Access which fosters wider collaboration and increased citations

- maximum visibility for your research: over $100 \mathrm{M}$ website views per year

At BMC, research is always in progress.

Learn more biomedcentral.com/submissions 